\title{
Programa Eureka en la resolución de problemas matemáticos en estudiantes de tercer grado de primaria
}

\author{
Using the Eureka Program for Solving \\ Mathematical Problems in Third-Grade Classes
}

\section{Uso do programa Eureka para solução de problemas matemáticos em classes de terceiro ano}

\author{
Sonia Vilma Sánchez Cosar* \\ Fecha de recepción: 10/10/2018 \\ Fecha de evaluación: 20/11/2018 \\ Fecha de aceptación: 25/11/2018 \\ Disponible en línea: \\ DOI: https://doi.org/10.18359/reds.4356
}

Cómo citar este artículo:

Sánchez, S. V. (2018). Programa Eureka en la resolución de problemas matemáticos en estudiantes de tercer grado de primaria. Revista Educación y Desarrollo Social, 12(2), 5-14. 


\title{
Resumen
}

El objetivo de esta investigación fue determinar si existían cambios notables al aplicar el programa Eureka en la resolución de problemas matemáticos en estudiantes de tercer grado de la Institución Educativa José Gálvez Barrenechea, en Tarma (Perú), durante 2017. El programa Eureka es un conjunto de actividades planificadas con el método de Pólya para la resolución de problemas matemáticos, considerando cuatro etapas: comprender el problema, elaborar un plan, ejecutar el plan y realizar una visión retrospectiva. En el estudio se empleó el método científico, experimental y estadístico.

Los resultados permitieron apreciar que los promedios variaron significativamente en el pre y el postest. En el pretest de resolución de problemas matemáticos el grado "3-B" obtuvo un puntaje de 12.96, el "3-C" logró 12.43 y el "3-D" alcanzó 12.05; mientras que en el postest de resolución de problemas matemáticos el GC (“3-B”) logró 13.00, el GE1 (“3-C") obtuvo 16.50 y el GE2 (“3-D”) consiguió 15.16. El programa Eureka influyó significativamente en la resolución de problemas, y se concluyó que hubo variaciones significativas en la resolución de problemas matemáticos, a través del Test ANOva con $\alpha=0.05$ de significancia, $\gamma=0.95$ de confianza, además $F$ calculada es mayor que F teórica $(20.65$ > 3.13), quedando demostrada la eficacia del programa Eureka.

Palabras clave: estrategias; programa Eureka; resolución de problemas matemáticos.

\begin{abstract}
This research project sought to determine whether noticeable changes occurred when third-grade students used the Eureka program for solving mathematical problems at the José Gálvez Barrenechea Educational Institution, in Tarma, Peru, in 2017. The Eureka program is a set of activities, based on the Pólya method for solving mathematical problems, distributed in four stages: understanding the problem, developing a plan, executing the plan, and performing a retrospective view. For this study, the scientific, experimental, and statistical methods were used. Since the pretest indicated that students in Class 3B reported better grades and higher-grade point averages than the other third-grade classes, this class was deemed as our control group (CG) while Classes 3C and 3D formed the experimental groups (EGl and EG2). Post test results denoted that the CG (Class 3B) achieved a score of 13.00, EG1 (Class 3C) obtained 16.50 and EG2 (Class 3D) scored 15.16. Therefore, the Eureka program did significantly influence problem solving. In fact, these significant variations in the resolution of mathematical problems were substantiated through an ANOVA test with a significance level of $\alpha=0.05$, a confidence level of $\gamma=0.95$, and where F calculated from the data was greater than the theoretical value of $\mathrm{F}(20.65>3.13)$, thus demonstrating the effectiveness of the Eureka program.
\end{abstract}

Keywords: strategies, Eureka program, mathematical problem solving.

\section{Resumo}

Este projeto de pesquisa procurou determinar se ocorreram mudanças perceptíveis quando alunos do terceiro ano usaram o programa Eureka para resolver problemas matemáticos na Instituição Educacional José Gálvez Barrenechea, em Tarma, Peru, em 2017. O programa Eureka é um conjunto de atividades, baseado no método Pólya para solução de problemas matemáticos, distribuído em quatro etapas: compreensão do problema, desenvolvimento de um plano, execução do plano e realização de uma visão retrospectiva. Para este estudo, foram utilizados os métodos científico, experimental e estatístico. Como o pré-teste indicou que os alunos da Classe 3B tiveram melhores notas e médias maiores do que as outras classes do terceiro ano, essa classe foi considerada como nosso grupo controle (GC) ao passo que as Classes 3C e 3D formaram os grupos experimentais (EG1 e EG2). Os resultados do pós-teste indicaram que o GC (Classe 3B) alcançou uma pontuação de 13,00, o EGl (Classe 3C) obteve 16,50 e o EG2 (Classe 3D) obteve 15,16. Portanto, o programa Eureka influenciou significativamente a solução de problemas. De fato, estas variações significativas na resolução de problemas matemáticos foram comprovadas através de um teste ANOva com um nível de significância de $\alpha=0.05$, um nível de confiança de $\gamma=0.95$, e onde $F$ calculado a partir dos dados foi maior que o valor teórico de $F(20,65>3,13)$, demonstrando assim a eficácia do programa Eureka.

Palavras-chave: estratégias, programa Eureka, resolução de problemas matemáticos. 


\section{Introducción}

El informe de Progreso Educativo de América Latina (2006), acerca de la participación de los países latinoamericanos en la evaluación PISA 2003, expone cómo los resultados obtenidos demostraron que muchos estudiantes no pueden aplicar en forma ordenada las habilidades matemáticas básicas para comprender y explorar situaciones contextualizadas. En la evaluación PISA 2015 Perú es el país que ha crecido más en América Latina, respecto a la medición de 2012; pese a que ha mejorado en ciencias, matemáticas y lectura, sigue rezagado, ubicándose en el puesto 64. En las evaluaciones nacionales ECE 2007 los estudiantes de segundo grado de primaria lograron en matemática el nivel satisfactorio con el $7.2 \%$ y después de diez años se ha logrado el $34.1 \%$. A pesar de que estos incrementos evidencian mejoras, se está distante de lo que debería lograrse, y los resultados siguen siendo menores en comparación a los de comprensión lectora.

Los resultados ECE 2016 de la UGEL 03, que corresponde a la provincia de Tarma, alcanzaron un $37.3 \%$. Estos resultados se han ido incrementando, pero son menores en comparación con la provincia de Yauli, que logró un nivel satisfactorio de $52.5 \%$.

La resolución de problemas es considerada en los últimos años como una actividad de suma importancia en el aprendizaje de la matemática, y se le considera como el eje vertebrador porque pone de manifiesto las capacidades de análisis, comprensión, razonamiento, aplicación y metacognición.

Las estrategias de enseñanza tienen la ventaja de promover aprendizajes significativos. Esto significa que el docente emplea estrategias para que el estudiante esté motivado para aprender con mayor interés. Anijovich y Mora (2012) refieren que las estrategias de enseñanza son un conjunto de decisiones que toma el docente para orientar la enseñanza, promover el aprendizaje significativo y crear nuevos conocimientos.

La teoría de Pólya (1981) está basada en el método heurístico para la solución de problemas matemáticos. Este método permite al estudiante ser protagonista de su propio aprendizaje, pues a través de la investigación y la experimentación descubre la solución de problemas, fomentando la responsabilidad e iniciativa y usando la creatividad y el pensamiento lateral o divergente.

El húngaro George Pólya plantea cuatro pasos que orientan la búsqueda de solución de problemas matemáticos:

- Comprender el problema. El estudiante debe comprender el problema, entender lo que se pide y, por lo tanto, plantearse interrogantes como: ¿de qué trata el problema?, ¿cómo lo diría con sus propias palabras?, ¿ha visto otra situación parecida?, ¿cuáles son los datos?, o ¿qué es lo que se pide? Estas interrogantes permiten movilizar sus saberes previos y es- 
tablecer relaciones sobre los datos del problema.

- Elaborar un plan. El estudiante comienza a explorar los caminos que puede seguir para resolver el problema. Por lo tanto, es necesario hacer las siguientes preguntas: ¿qué deberíamos hacer primero?, ¿debemos considerar todos los datos?, ¿cómo haríamos para llegar a la respuesta?, ¿se ha resuelto algún problema parecido? Es necesario contar con un conjunto de estrategias heurísticas para potenciar los conocimientos de los estudiantes. Ministerio de Educación del Perú (2013) sugiere algunas estrategias heurísticas: hacer simulaciones, buscar patrones, ensayo-error, hacer un diagrama, usar analogías y empezar por el final.

- Ejecución del plan. Es el proceso en el que el estudiante deberá aplicar el plan, empleará conocimientos adquiridos y hará uso de habilidades del pensamiento y la concentración. Es necesario plantear estas interrogantes: ¿considera que los procedimientos ayudarán a encontrar la respuesta?, ¿habrá otros caminos para hallar la respuesta?, ¿está seguro de la respuesta?, ¿cómo lo comprueba? Estas preguntas hacen que los estudiantes desarrollen sus estrategias, comprueben sus resultados y, si no se tiene éxito, se vuelve a empezar.

- Visión retrospectiva. El estudiante vuelve a examinar la estrategia que empleó, así como la solución y el resultado. La práctica permitirá fortalecer sus conocimientos e incluso mejorará su comprensión de la solución a la cual llegó. Es preciso preguntarse: ien qué se parece este problema a otros?, ¿cómo se hizo para hallar la respuesta?, ¿qué dio la pista para elegir la estrategia?, ¿fue difícil resolver el problema? (ipor qué?), ¿cree que el material utilizado ayudó? (ipor qué?). Este paso debe ser aprovechado por el docente para que el estudiante constate la relación del problema resuelto con otro que pudiera requerir un razonamiento más o menos similar, e inclusive pueda aplicarlo en la solución de problemas de la vida cotidiana.

El propósito del método es que la persona examine y construya su propio método de pensamiento de manera sistemática, eliminando obstáculos para establecer hábitos mentales eficaces, a lo que Pólya denominó pensamiento productivo. En tal sentido, la investigación se planteó los siguientes objetivos: determinar si existen diferencias significativas al aplicar el programa Eureka en la resolución de problemas matemáticos en estudiantes del tercer grado de primaria de la Institución Educativa José Gálvez Barrenechea de Tarma, Perú, en 2017; y aplicar la resolución de problemas matemáticos de estructura aditiva de una etapa, de estructura aditiva de dos etapas, y de estructura multiplicativa.

A continuación se detallan los antecedentes de proyectos similares: Medina (2013), en su investigación Influencia del método heurístico para la enseñanza-aprendizaje de 
la matemática en alumnos del tercer grado de secundaria del distrito de Cajabamba, tuvo por objetivo determinar la eficacia del método heurístico en la enseñanza-aprendizaje del área de matemática (ecuaciones). La muestra estuvo conformada por 30 estudiantes de la Institución Educativa José Gálvez Egúsquiza (grupo control) y 29 estudiantes de la Institución Educativa José Sabogal Diéguez (grupo experimental). Utilizó el cuestionario y el método de una prueba previa y otra posterior. Concluyó que la aplicación del método heurístico incrementa el rendimiento académico en el área de matemática de los alumnos de tercer grado de secundaria.

Y Llatas (2016), en el estudio Programa de estrategias metodológicas para mejorar las habilidades matemáticas de los estudiantes del IsEP Octavio Matta Contreras de Cutervo, quiso demostrar que la aplicación de un programa de estrategias metodológicas mejora las habilidades matemáticas de los estudiantes. Fue una investigación explicativa, aplicada con un diseño pre-experimental. La muestra estuvo conformada por 76 estudiantes de segundo semestre de las especialidades de Educación Inicial, Idioma Inglés y Matemáticas. Los instrumentos que utilizó para el estudio fueron la lista de cotejo y el test (pre y pos). Concluyó que la aplicación del programa de estrategias metodológicas ha resultado lo suficientemente válido para los estudiantes del ISEP Octavio Matta Contreras, de Cutervo, pues constituyó una alternativa eficaz para mejorar el nivel de desarrollo de las habilidades matemáticas.
La presente investigación se justificó por:

a) Conveniencia. Abordó un tema de significatividad pedagógica que influyó en la resolución de problemas matemáticos con la aplicación del método de Pólya a través del programa Eureka, enseñando al estudiante a matematizar y aumentar su confianza, volviéndose más perseverante y creativo y mejorando su espíritu investigador. En el ámbito de la didáctica, permitió que el docente conociera y aplicara la propuesta al ser una metodología ordenada y sistemática.

b) Relevancia social. Contribuyó con los fines de la educación y respondió a los propósitos de la Educación Básica Regular al año 2021 (Ministerio de Educación del Perú, 2009). Asimismo, fue relevante para la Institución Educativa José Gálvez Barrenechea, pues permitió mejoras del aprendizaje en los educandos y de la calidad educativa.

c) Implicancias prácticas. Es conocido que los docentes trabajan con los estudiantes ejercicios rutinarios y mecánicos que están lejos de estimular los procesos cognitivos, y el estudio permitió a los docentes implementar metodologías eficaces e incrementar el pensamiento matemático de los estudiantes para que pudieran enfrentar las evaluaciones, que constituyen un reto tanto para los estudiantes como para los profesores. 
d) Valor teórico. El resultado de este estudio se vincula al campo de la teoría del conocimiento, pues aporta información relevante sobre problemas matemáticos y estrategias de enseñanza, como el método de Pólya. Las variables de estudio tienen sustento teórico en el enfoque cognitivo; al resolver problemas matemáticos el estudiante hace uso de las operaciones mentales. También ratificó los fundamentos de la teoría constructivista, fundamentados en que al estudiante se le debe ayudar a generar andamios cognoscitivos y crear su propio conocimiento.

e) Utilidad metodológica. Para el estudio se elaboró el instrumento "Prueba de resolución de problemas matemáticos", que permitió medir problemas de estructura aditiva de una etapa, de estructura aditiva de dos etapas y de estructura multiplicativa, y el cual pasó por un "juicio de expertos", empleándose como pretest y postest. El programa Eureka aplicó el método de Pólya con cuatro pasos: comprender el problema, diseñar un plan, ejecutar el plan y examinar la solución. Demostró así la validez y confiabilidad del instrumento y la eficiencia del programa; entonces pudo ser utilizado en otros trabajos de investigación.

\section{Materiales y métodos}

10 El método de investigación fue aplicado y correspondió al nivel experimental, donde se suministró el programa Eureka con los pasos de Pólya para mejorar la resolución de problemas matemáticos en los estudiantes del tercer grado de primaria. La investigación corresponde al diseño cuasi experimental con pre y postest con grupo experimental y grupo control. El diseño responde al siguiente esquema:

$$
\begin{array}{cccc}
\mathrm{GE}_{1} & \mathrm{O}_{1} & \mathrm{X} & \mathrm{O}_{4} \\
\mathrm{GE}_{2} & \mathrm{O}_{2} & \mathrm{X} & \mathrm{O}_{5} \\
\hdashline & & & \\
\mathrm{GC}_{3} & \mathrm{O}_{3} & - & \mathrm{O}_{6}
\end{array}
$$

Donde:

$\mathrm{GE}_{1}=$ Grupo Experimental 1

$\mathrm{GE}_{2}=$ Grupo Experimental 2

$\mathrm{GC}=$ Grupo Control

$\mathrm{O}_{1}, \mathrm{O}_{2}$ y $\mathrm{O}_{3}=$ Pretest de resolución de problemas

$\mathrm{X}=$ Programa Eureka

$\mathrm{O}_{4} \mathrm{O}_{5}$ y $\mathrm{O}_{6}=$ Postest de resolución de problemas

Se utilizó el método científico como método universal, lo que permitió un mejor planteamiento y formulación del problema respecto a la aplicación del programa en la resolución de problemas. Asimismo, se aplicaron el método experimental y el método estadístico.

La población estuvo conformada por 157 estudiantes de tercer grado de primaria 
de la Institución Educativa José Gálvez Barrenechea (N. $\left.{ }^{\circ} 31518\right)$, matriculados en el año 2017. La muestra determinada fue de 24 estudiantes del grado " $3-\mathrm{B}$ ", 28 del "3-C" y 19 del grado "3-E".

El instrumento "Prueba de resolución de problemas matemáticos", aplicada a los estudiantes del tercer grado, es una prueba objetiva de opción múltiple, que permitió medir el nivel de logro en la resolución de problemas. Guarda relación con el Currículo Nacional y toma en cuenta la competencia y la capacidad del IV ciclo de la EBR.

La prueba consta de 20 problemas con 4 posibilidades de respuestas cada uno a través de problemas aditivos de una etapa, aditivos de dos etapas y multiplicativos. La aplicación de la prueba puede ser individual o colectiva, con un tiempo aproximado de 60 minutos y se puede aplicar con grupos de hasta 30 estudiantes.

La calificación se realizó asignando un punto por cada respuesta correcta y cero puntos por cada incorrecta. El puntaje se obtuvo sumando las respuestas correctas y convirtiendo el puntaje a una calificación literal en base a la escala de calificación de los aprendizajes en EBR, propuesto por el Ministerio de Educación del Perú (2009). El puntaje va de 0 a 20 puntos. Para determinar la validez del instrumento se consideró el "juicio de expertos", utilizando el Coeficiente V. de Aiken, puesto que los valores son contrastados estadísticamente según el tamaño de la muestra de los jueces consultados. Este coeficiente puede obtener valores entre 0 y 1 , a medida que sea más elevado el valor computado, el ítem tendrá una validez de contenido. Se aplicó la prueba piloto a 24 estudiantes para medir la confiabilidad, empleándose el coeficiente de Kuder-Richardson y arrojando el valor de 0.8092 .

Para el procedimiento de recogida de datos la técnica empleada fue la prueba o examen. El instrumento utilizado fue la "Prueba de resolución de problemas matemáticos", que nos permitió recoger información sobre la resolución de problemas matemáticos y se aplicó en dos momentos: pretest y postest al grupo control y al experimental. Asimismo, para la ejecución de la investigación se llevó el oficio de autorización emitido por la Universidad César Vallejo a la Institución Educativa José Gálvez Barrenechea (N. ${ }^{\circ} 31518$ ), el personal directivo autorizó la aplicación del programa Eureka y la aplicación de la "Prueba de resolución de problemas matemáticos" y se protegió el anonimato de cada estudiante respecto al nivel alcanzado.

\section{Resultados}

Con el objetivo de evaluar la resolución de problemas matemáticos en estudiantes de tercer grado, se diseñó un programa con un conjunto de actividades y se les evaluó con la "Prueba de resolución de problemas matemáticos" mediante un pretest $\mathrm{y}$ un postest. 
Tabla 1. Resultados del pretest de resolución de problemas matemáticos aplicado en tercer grado

\begin{tabular}{|l|l|l|l|l|}
\hline & "3-B" & "3-C" & "3-D" & Relación \\
\hline X & 12.96 & 12.43 & 12.05 & $12.96>12.43>12.05$ \\
\hline Me & 13.00 & 12.50 & 13.00 & $13>12.50<13$ \\
\hline Mo & 13.00 & 13.00 & 13.00 & $13=13=13$ \\
\hline S & 3.61 & 7.59 & 4.61 & $3.61<7.59>4.61$ \\
\hline S & 1.90 & 2.75 & 2.15 & $1.90<2.75>2.15$ \\
\hline CV & $14.66 \%$ & $22.16 \%$ & $17.81 \%$ & $14.66 \%<22.16 \%>17.81 \%$ \\
\hline
\end{tabular}

Fuente: elaboración propia a partir de los resultados de la prueba.

En la tabla 1, después de la aplicación del pretest de resolución de problemas en la sección "3-B", los resultados muestran que tiene mayor promedio con relación a las otras secciones (12.96>12.43> 12.05). Respecto a la mediana, los estudiantes de "3-B" y "3-E" tienen como calificativol3, en comparación con "3-C", que obtuvo 12.50. Respecto a la moda, las tres secciones presentan frecuencia de 13. Así mismo, los estudiantes del grado "3-B" obtuvieron menor desviación estándar con respecto a las otras secciones
$(1.90<2.75>2.15)$. También el grado "3B" obtuvo el menor coeficiente de variación en comparación con las otras secciones ( $14.66 \%>22.78 \%>17.81 \%)$, llegando a la conclusión de que los estudiantes de este grado han obtenido mayor promedio y reflejan mayor homogeneidad en los resultados de las tres secciones. Asi se determinó como grupo control en la investigación y los otros grupos formaron parte del grupo experimental.

Tabla 2. Resultados del postest de resolución de problemas matemáticos aplicado en los grupos experimental y de control

\begin{tabular}{|l|l|l|l|l|}
\hline & $\mathrm{GE}_{1}$ & $\mathrm{GE}_{2}$ & $\mathrm{GC}$ & Relación \\
\hline $\mathrm{X}$ & 16.50 & 15.16 & 13.00 & $16.50>15.16>13.00$ \\
\hline $\mathrm{Me}$ & 16.00 & 15.00 & 13.00 & $16>15>13$ \\
\hline $\mathrm{Mo}$ & 16.00 & 15.00 & 13.00 & $16>15>13$ \\
\hline $\mathrm{S}^{2}$ & 2.41 & 6.36 & 3.91 & $6.36_{2}>3.91>2.41_{1}$ \\
\hline $\mathrm{S}$ & 1.55 & 2.52 & 1.98 & $2.52_{2}>1.98>1.55_{1}$ \\
\hline $\mathrm{CV}$ & 9.40 & 16.64 & 15.22 & $\begin{array}{l}9.40_{1}<15 \\
22_{1}<16.64_{2}\end{array}$ \\
\hline Asimetría & 0.48 & 0.13 & -0.29 & $0.48_{2}<0.13_{1}<-0.29$ \\
\hline Curtosis & 0.87 & 0.39 & -0.80 & $0.87_{1}>0.39_{2}>-0.80$ \\
\hline
\end{tabular}

Fuente: elaboración propia a partir de los resultados de la prueba. 
En la tabla 2 se ve que en el postest de resolución de problemas los estudiantes del $\mathrm{GE}_{1}$ lograron un promedio de 16.50 puntos en comparación con el $\mathrm{GE}_{2}$, que obtuvo 15.16 puntos, y el grupo control, que obtuvo 13 puntos. Respecto a la mediana, el GE 1 obtuvo 16 puntos, el $\mathrm{GE}_{2}$ alcanzó 15 puntos, mientras que el GC consiguió 13 puntos. Con relación a la moda, los estudiantes del $\mathrm{GE}_{1}$ presen$\tan 16$ puntos, los estudiantes del $\mathrm{GE}_{2}$ muestran 15 puntos y los estudiantes del GC ostentan 13 puntos. Referente a la varianza, las notas del grupo GC son de 3.91 y son mayores en comparación con la variabilidad de las notas del $\mathrm{GE}_{1}$, que presenta 2.41, pero menor que del $\mathrm{GE}_{2}$, que muestra 6.36; esto significa $6.36_{2}>3.91>2.41_{1}$. Asimismo, la desviación estándar del GC se dispersa en promedio 1.98 puntos alrededor de la media aritmética de 13 , y es mayor en comparación a la desviación del $\mathrm{GE}_{1}$, que se dispersa en promedio 1.55 puntos alrededor de la media aritmética de 16.50 , pero menor a la desviación del $\mathrm{GE}_{2}$, que se dispersa en promedio 6.36 puntos alrededor de la media aritmética de 15.16. En el coeficiente de variación en los grupos experimental y de control observamos una homogeneidad en los datos, porque son menores al $33 \%$. Al comparar estas evidencias $\left(9.40_{1}<15.22<16.64_{2}\right)$, el GE 1 es más homogéneo pues los datos de la distribución presentan menor dispersión. En el coeficiente de asimetría, el GE y el GC presentan una asimetría positiva y un sesgo hacia la izquierda, lo que indica que el contenido de la prueba estuvo difícil. Mientras que el $\mathrm{GE}_{2}$ presenta una asimetría negativa y un sesgo hacia la derecha, lo que significa que la prueba estuvo fácil.

\section{Discusión}

Los resultados del pretest de resolución de problemas matemáticos nos indican que los estudiantes de los grados "3-A", "3-B" y "3-E" lograron promedios de 12.96, 12.43 y 12.05 , ubicándose en el nivel de inicio (00-10) y proceso (11-13); en consecuencia, los estudiantes presentan dificultades para solucionar problemas matemáticos aditivos y multiplicativos debido a diversos factores, como la falta de estrategias de enseñanza y la dificultad para la comprensión lectora del estudiante al realizar el análisis de los enunciados.

Silva (2016), en su investigación Relación entre el nivel de comprensión lectora y resolución de problemas matemáticos en los estudiantes de primer ciclo de la carrera de Topografía en la Escuela Superior Tecnológica Sencico (Lima), afirma que la comprensión lectora es un proceso cognitivo e interactivo, y considera que es un diálogo entre el lector y el autor del texto. El resultado logrado en dicha investigación fue desfavorable, pues los estudiantes presentaron un nivel deficitario del $70 \%$ en comprensión lectora.

Lessenger, Wilson y Engelhart explican que, si se mejora la capacidad lectora, aumenta la capacidad para resolver problemas verbales y se van desarrollando las habilidades matemáticas. 
Respecto a la hipótesis general, los resultados del postest demostraron que existe diferencia significativa de los promedios al comparar $\mathrm{GE}_{1}, \mathrm{GE}_{2}$ y $\mathrm{GC}$, por medio de análisis de varianza (ANOVA) donde $F_{c}$ es mayor a $F_{t}(20.65>3.13)$, entonces se rechazó la hipótesis nula y se aceptó la hipótesis alterna.

La conclusión fue que existe una diferencia significativa de los promedios previos y posteriores a la aplicación del programa Eureka en la resolución de problemas matemáticos en estudiantes de tercer grado de la Institución Educativa José Gálvez Barrenechea, en Tarma, Perú, en 2017. Más aun, la prueba de Tukey-Snedecor permitió determinar la diferencia entre los grupos $\mathrm{GE}_{1}, \mathrm{GE}_{2}$ y GC, donde $\mathrm{D}<\mathrm{D}_{\mathrm{GEI}-\mathrm{GC}}$, porque $1.28<3.50$; existiendo diferencia significativa entre los puntajes de estos grupos. Además, $\mathrm{D}<\mathrm{D}_{\mathrm{GE2} 2 \mathrm{GC}}$, porque $1.28<2.00$; es decir, existe una diferencia significativa entre los puntajes de estos grupos. También $\mathrm{D}<\mathrm{D}_{\mathrm{GE1}-\mathrm{GC} 2}$, porque $1.28<1.50$; es la diferencia entre $\mathrm{GE}_{1}$ y $\mathrm{GE}_{2}$, es decir que existió una diferencia significativa entre los puntajes de estos grupos.

Finalmente, la aplicación del programa Eureka influyó significativamente en la resolución de problemas matemáticos en estudiantes de tercer grado de la Institución Educativa José Gálvez Barrenechea. Se concluyó que existen diferencias significativas porque al haber evaluado la

14 hipótesis general a través de Test AnOva con de significancia de confianza, además F calculada es mayor a F teórica (20.65 $>3.13$ ); en consecuencia, rechazamos la hipótesis nula (H0) y aceptamos la hipótesis alterna $(\mathrm{Ha})$.

\section{Referencias}

Anijovich, R. y Mora, S. (2012). Estrategias de enseñanza. Buenos Aires: Aique.

Llatas, M. (2016). Programa de estrategias metodológicas paramejorar las habilidades matemáticas de los estudiantes del ISEP Octavio Matta Contreras de Cutervo. [Tesis de doctorado]. Lima: Universidad César Vallejo.

Medina, N. (2013). Influencia del método heurístico paralaenseñanza-aprendizaje de la matemática en alumnos del tercergrado de secundaria del distrito de Cajabamba. [Tesis de doctorado]. Trujillo, Perú:Universidad Privada Antenor Orrego de Trujillo.

Ministerio de Educación del Perú. (2009). Diseño Curricular Nacional de la Educación Básica Regular. Lima: World Color Perú. Ministerio de Educación del Perú. (2013). Rutas de aprendizaje. Lima: Corporación Gráfica Navarrete.

Pólya, G. (1981). ¿Cómo plantear y resolver matemática? México: Trillas.

Progreso Educativo de América Latina [PREAL]. (2006). Programa de Promoción de la Reforma Educativa en América Latina y el Caribe. "Cantidad sin Calidad". Washington, D. C.: PREAL.

Silva, B. (2016). Relación entre el nivel de comprensión lectora y la resolución de problemas matemáticos en los estudiantes del primer ciclo de la carrera de Topografía en la Escuela Superior Tecnológica Sencico, sede Lima (2014). Lima: Sencico. 